\title{
Protective effects of the traditional herbal formulation on oxidative stress, learning and memory in the animal model of type 2 diabetes
}

\author{
Dara Dastan ${ }^{1,2}$, Iraj Salehi ${ }^{3 *}$ iD, Alireza Komaki ${ }^{3}$, Alireza Gharib ${ }^{3}$, Alireza Pouyandeh Ravan ${ }^{4}$, Ali \\ Mohebi Zadeh $^{3}$, Nafiseh Faraji ${ }^{3}$, Nejat Kheiripour ${ }^{5 *}$ iD \\ 1. Medicinal Plants and Natural Products Research Center, Hamadan University of Medical Sciences, Hamadan, Iran \\ 2. Department of Pharmacognosy and Pharmaceutical Biotechnology, School of Pharmacy, Hamadan University of Medical \\ Sciences, Hamadan, Iran \\ 3. Neurophysiology Research Center, Hamadan University of Medical Sciences, Hamadan, Iran \\ 4. Department of Medical Laboratory Sciences, School of Para-Medicine, Hamadan University of Medical Sciences, \\ Hamadan, Iran \\ 5. Research Center for Biochemistry and Nutrition in Metabolic Diseases, Kashan University of Medical Sciences, Kashan, \\ Iran
}

\section{Abstract}

Introduction: Diabetes mellitus (DM) is one of the most frequent metabolic diseases that affect various body systems. Cognitive impairment caused by diabetes is gaining more acceptance and attention. In this study, we have investigated the effects of a traditionally herbal formulation (THF) on oxidative stress (OS) and cognitive deficits in type 2 diabetic rats.

Methods: Thirty-six male Wistar rats were divided into six groups: control group, diabetic group, diabetic $+100,200$ or $300 \mathrm{mg} / \mathrm{kg} \mathrm{THF}$, diabetic+glibenclamide (G) $5 \mathrm{mg} / \mathrm{kg}$. Streptozotocin-nicotinamide was used to induce type-II diabetes mellitus. Spatial and passive avoidance learning and memory function were evaluated by Morris Water Maze (MWM), novel object recognition test (NORT) and open field test (OFT). The OS biomarkers were also analyzed. The THF was standardized using RPHPLC according to phenolic and flavonoids compounds.

Results: Indicated that in the diabetic treated $(300 \mathrm{mg} / \mathrm{kg}$ THF and G) vs. diabetic groups, body weight and insulin were significantly increased and the levels of fasting blood glucose significantly reduced. OS was improved in the treated $(300 \mathrm{mg} / \mathrm{kg} \mathrm{THF})$ groups. Furthermore, we noticed that diabetic treated groups $(300 \mathrm{mg} / \mathrm{kg}$ THF) vs. diabetes caused in significant decreases of the travelled distance and escape latency to find the hidden platform, also increased in the time spent and travelled distance in the target quadrant in MWM test, exploration time in NORT and total distance moved in OFT.

Conclusion: These findings suggest that THF ameliorated learning and memory deficits in type 2 diabetic rats via reducing OS. THF can be used with a caution against human DM.

\section{Keywords:}

Medicinal Herbs;

Type 2 diabetes;

Learning and Memory;

Oxidative Stress;

Morris Water Maze;

\section{* Corresponding author:}

N. Kheiripour

Email:

kheiripour-n@kaums.ac.ir

I. Salehi

Email:

salehi@umsha.ac.ir

Tel: +98 (811) 8380462-4

Received 10 August 2019;

Received in revised form 1

April 2020; Accepted 4 May

2020 


\section{Introduction}

Diabetes mellitus (DM) is a chronic metabolic disease and is major public health problems in the world and studies showed that 592 million worldwide will suffer DM by 2035 (Guariguata et al., 2014). The prevalence of dementia and cognitive dysfunction is frequently observed in DM patients (Kodl and Seaquist, 2008; Zarrinkalam et al., 2017). The mechanisms underlying the development of cognitive dysfunction in DM have not been fully elucidated. Recently recognized that apoptosis, neuron degeneration and oxidative stress are significantly raised in the hippocampus of animal models of DM (Seto et al., 2015). The oxidative stress imposed by reactive oxygen species plays an important role in many chronic and degenerative diseases (Ardjmand et al., 2019; Goli et al., 2019).

Studies suggested that antidiabetic drugs could offer therapeutic benefits to DM-related impairment of cognitive functioning in both patients and animal models (Risner et al., 2006). On the other hand, these drugs frequently exerted side effects, including bone loss, weight gain and increased risk of cardiovascular events. These side effects could become more prevalent due to continuous use. Furthermore, treatment is very costly as well, since type 2 diabetes mellitus (T2DM) is a chronic disease and long-term medications are necessary ( $\mathrm{Li}$ et al., 2004). Traditional herbal medicine (THM) can be a good alternative to replace or at least supplement to medications. A large number of THM has been used to treat T2DM complications for over thousands of years (Wang et al., 2013). THM treating T2DM can target multiple mechanisms such as raised insulin secretion and sensitivity, or decreased of carbohydrate absorption, anti-inflammatory and exert antioxidant effects (Li et al., 2004; Rahimi et al., 2018). Results from T2DM mouse model studies show that glycemic control using natural products and herbal extracts significantly prevented cognitive impairment via attenuation of cerebral oxidative stress and increase in cerebral brain-derived neurotrophic factor (Seto et al., 2015). THM contains oligomeric proanthocyanidins, flavonoids and polyphenols, which are well-known for their antioxidant, anti-inflammatory and antidiabetic properties (Ravan et al., 2017; Rios et al., 2015). Since the natural products and THM is relatively safe and have fewer side effects compared to other antidiabetic drugs, in this study we uses a combination of various herbs (cinnamon, nettles, Achillea millefolium [Yarrow], Arctium lappa, Portulaca oleracea, Acroptilon repens, Sativus crocus, Walnut and Rhus coriaria [Sumac)]) that are medically important and have great use in traditional medicine. Studies show that the mentioned plants have a high content of phenols and flavonoids such as gallic acid, benzoic acid, naringenin, rutin, quercetin and apigenin (Mansouri et al., 2013; Salehi et al., 2018).

Therefore, the purpose of this article was to examine the effect of the THF on impaired learning and memory function and oxidative stress indices in T2DM rats

\section{Materials and methods \\ Chemicals and drugs}

Streptozotocin, nicotinamide, thiobarbituric acid, ferric chloride hexahydrate, n-butanol and sodium dodecyl sulfate were purchased from the Sigma Chemical Co. (United States). Nitric acid, perchloric acid and acetic acid (glacial) were purchased from Merck (Germany). All the other chemicals used were of analytical grade.

\section{Plant extraction}

The plants were purchased from the market and voucher samples were deposited at the Herbarium of the Faculty of Pharmacy, Hamadan University of Medical Sciences, Hamadan, Iran. About $100 \mathrm{~g}$ of powdered plants were extracted with $500 \mathrm{ml}$ of distilled water for $48 \mathrm{~h}$ using the maceration method and a deep brown aqueous extract was obtained. The extract was dried using a rotary evaporator and freeze drying and finally stored in universal bottles and refrigerated at $4^{\circ} \mathrm{C}$ prior to using. The dried substance was dissolved in normal saline $(\mathrm{pH} 7.4)$. Animals were treated with 100,200 or $300 \mathrm{mg} / \mathrm{kg}$ body weight of the THF, by gavage every day for 60 days.

\section{Standardization of herbal formulation by RP- HPLC-PDA}

Standardization of herbal formulation was done according to phenolic and flavonoids by RP-HPLCPDA. The quantitative analysis was performed with external standardization by measurement of the peak areas using LabSolutions (Shimadzu) software. Seven different concentrations of ferulic acid, gallic 


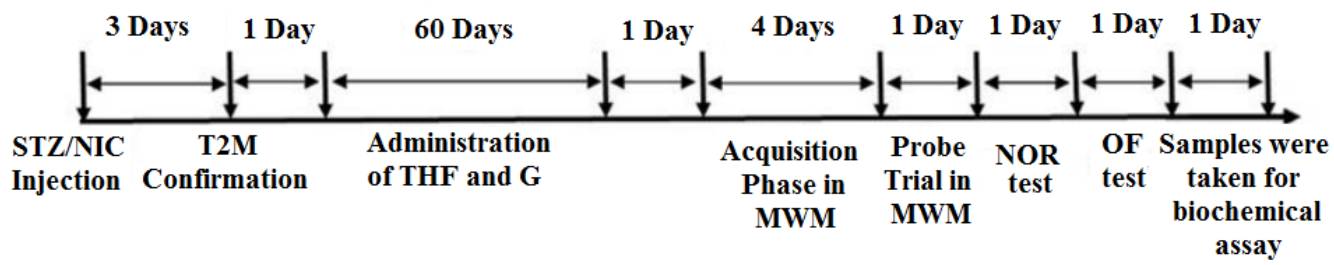

Fig.1. Experimental timeline. Type 2 diabetes mellitus model was induced by intraperitoneal injection of streptozotocinnicotinamide, and confirmed by a fasting blood sugar level of $>126 \mathrm{mg} / \mathrm{dl} 3$ days later. Administration of traditional herbal formulation (THF) in 100, 200 and $300 \mathrm{mg} / \mathrm{kg}$, and glibenclamide $5 \mathrm{mg} / \mathrm{kg}$ began 1 day after confirmation of T2DM, by gavage every day for 60 days. For measurement of learning and memory Morris water maze (MWM), novel object recognition (NOR) and open field (OF) test were used. Also, oxidative stress parameters in the hippocampus, FBS and serum insulin was measured.

acid, caffeic acid, benzoic acid, naringenin, rutin, quercetin and apigenin (Sigma-Aldrich) were used for the calibration curve. The HPLC column was a Spherisorb ODS-2 $(5 \mu \mathrm{m})$ reversed phase $4.6 \mathrm{~mm} \times$ $250 \mathrm{~mm}$ and the flow rate of mobile phase $(\mathrm{MeOH}$ in $\mathrm{H}_{2} \mathrm{O} /$ acetic acid $(5-100 \% \mathrm{MeOH})$ was carried out at a $1 \mathrm{ml} / \mathrm{min}$. Different parameters including UV spectra, retention times and comparison with phenolic and flavonoid standards were used for the identification of compounds.

\section{Animals and experimental designs}

Thirty-six male Wistar rats $(220 \pm 20 \mathrm{~g})$ were housed under standard conditions (12h dark/light cycle at $\left.22 \pm 2^{\circ} \mathrm{C}\right)$. Animals were divided into 6 groups $(n=6)$ : group $\mathrm{C}$, normal control; group $\mathrm{D}$, diabetic control; diabetic rats treated with THF $(100,200$ or $300 \mathrm{mg} / \mathrm{kg}$ and glibenclamide [G] $5 \mathrm{mg} / \mathrm{kg}$ respectively for 60 days). All procedures of this study were approved by the Medical Ethics Committee of Hamadan University of Medical Sciences (IR.UMSHA.REC.1396.101).

For induction of T2DM, $15 \mathrm{~min}$ after the injection of nicotinamide (120mg/kg; ip), streptozotocin $(60 \mathrm{mg} / \mathrm{kg}$; ip) dissolved in $0.1 \mathrm{M}$ of citrate buffer $(\mathrm{pH} \mathrm{4.5)}$ was injected in fasted rats. To confirm the T2DM, after $72 \mathrm{~h}$ fasting blood sugar (FBS) level of rats was measured with a glucometer (Accuchek; Roche, Germany), the animals were considered diabetic when FBS level of $>126 \mathrm{mg} / \mathrm{dl}$ (Shirwaikar et al., 2006). At the end of experiments, under ether anaesthesia blood samples were taken from the inferior vena cava, serum was separated and stored at $-20^{\circ} \mathrm{C}$ for biochemical analysis. Also, the hippocampus tissue was collected from each rat and cleaned with an ice-cold saline solution and frozen in liquid nitrogen immediately after separation and stored at $-70^{\circ} \mathrm{C}$ until analysis. The experimental timeline is shown in Figure 1.

\section{Assay of serum insulin and hippocampus oxidative stress parameters}

Insulin concentration was determined using the rat insulin ELISA Kit (Mercodia, Uppsala, Sweden). The amount of hippocampus lipid peroxidation was predicted as the concentration of thiobarbituric acid reactive output malondialdehyde (MDA) according to Yagi's method (Zanganeh et al., 2018). Thiobarbituric acid reacts with MDA and is formed thiobarbituric acid reactant substances, as biomarkers of oxidative damage to polyunsaturated fatty acids. Total antioxidant capacity (TAC) determined by Benzi and Strain method. Hippocampus TAC was measured using the ferric reducing ability of serum which is a test shows antioxidant capacity and quantifies the ability of serum to reduce ferric ion to ferrous ion (Benzie and Strain, 1996). Hippocampus total thiol molecules (TTM) were determined using Ellman's reagent (DTNB; 5,5'-dithio-bis-[2-nitrobenzoic acid]) according to the $\mathrm{Hu}$ method and total oxidant status (TOS) of liver samples was determined by the ferricxylenol orange 1 reagent, according to the method described by Erel, also the oxidative stress index (OSI) was calculated by dividing TOS/TAC (Kheiripour et al., 2019).

\section{Behavioral tests}

\section{Morris Water Maze (MWM)}

The MWM was used to measure the ability of spatial learning and memory. In brief, the water maze was a circular tank $180 \mathrm{~cm}$ in diameter, $60 \mathrm{~cm}$ in height, black coloured, filled to a depth of $25 \mathrm{~cm}$ with water at $22 \pm 1^{\circ} \mathrm{C}$ ) located in a room containing a variety of 
Table 1: The effect of THF on rats' body weight, FBS and insulin in different studied groups

\begin{tabular}{|c|c|c|c|c|c|c|}
\hline $\begin{array}{c}\text { Parameters / } \\
\text { Group }\end{array}$ & C & D & $\begin{array}{c}D+100 \\
\mathrm{mg} / \mathrm{kg} \text { THF }\end{array}$ & $\begin{array}{c}\mathrm{D}+200 \mathrm{mg} / \mathrm{kg} \\
\text { THF }\end{array}$ & $\begin{array}{c}\mathrm{D}+300 \mathrm{mg} / \mathrm{kg} \\
\text { THF }\end{array}$ & $\mathbf{D}+\mathbf{G}$ \\
\hline $\begin{array}{l}\text { Final body } \\
\text { weight (g) }\end{array}$ & $265.50 \pm 12.51$ & $190.67 \pm 8.28^{\mathrm{a}^{*}}$ & $200.00 \pm 9.40$ & $210.33 \pm 10.34$ & $257.78 \pm 11.03^{b^{\star}}$ & $226.50 \pm 21.78^{b^{*}}$ \\
\hline FBS (mg/dl) & $75.50 \pm 6.44$ & $292.26 \pm 16.19^{\mathrm{a}^{*}}$ & $269.50 \pm 18.49$ & $253.67 \pm 17.50^{\text {b十 }}$ & $79.33 \pm 11.11^{\mathbf{b}^{*}}$ & $207.17 \pm 17.88^{b^{*}}$ \\
\hline Insulin $(\mu \mathrm{U} / \mathrm{ml})$ & $12.05 \pm 1.54$ & $6.44 \pm 0.49^{\mathrm{a}^{*}}$ & $7.09 \pm 0.66$ & $7.35 \pm 0.57$ & $12.51 \pm 0.0516^{\mathbf{b}^{*}}$ & $8.13 \pm 0.71^{b^{*}}$ \\
\hline \multicolumn{7}{|c|}{$\begin{array}{l}\text { Results were expressed as mean } \pm \text { SD. C: control; D: diabetic; THF: traditional herbal formulation; G: glibenclamide } \\
5 \mathrm{mg} / \mathrm{kg} \text {; FBS: fasting blood sugar. }{ }^{a} \text { significantly compared to the healthy control group. }{ }^{b} \text { significantly compared to the } \\
\text { diabetic control group. }{ }^{\dagger} P<0.05,{ }^{\ddagger} P<0.01 \text { and }{ }^{\star} P<0.001 \text {. }\end{array}$} \\
\hline
\end{tabular}

visual cues. Low light was used for illumination and the room was sound insulated. The pool had four quadrants with four starting lines named north $(\mathrm{N})$, east $(E)$, south $(S)$ and west (W) and an invisible Plexiglas platform $(10 \mathrm{~cm}$ in diameter) centrally located $1 \mathrm{~cm}$ beneath the water in quadrant $\mathrm{N}$. In our studies, 4-day training trials of animals were conducted at nearly the same time and each day had three blocks with four trials (90s). If an animal did not escape within 90s, it was manually guided to the escape platform by the experimenter. There was a 20s gap between three trials on the platform and the rest time was $5 \mathrm{~min}$ between two consecutive blocks. Twenty-four hours after 4th training test, the probe test was performed in which the platform was removed from the pool and the rat was allowed to swim for 60s while we recorded the ratio of time spent in the target quadrant and the swimming speed (Asadbegi et al., 2017). All trials were processed online by a video track tracking system. The escape latency, the time spent in the target quadrant and the times crossing the platform were used to evaluate the animals spatial learning and memory ability (Zarrinkalam et al., 2017).

\section{Novel object recognition test (NORT)}

The NORT is used extensively to investigate the visuospatial memory of animals in a familiar environment. Twenty-four hours before the test, each of the rats was placed in an individual apparatus used for object recognition tasks $(70 \times 50 \times 40 \mathrm{~cm})$ for $20 \mathrm{~min}$ for acclimatization so that their exploratory behavior does not interfere with their interaction with objects. The next day, two identical objects (round or square bowls) were placed in the box, following which each rat was placed alone at the midpoint as well as next to the front wall of the box opposite the objects. They were allowed to explore the objects for $10 \mathrm{~min}$ and were then taken back to the cages (familiarization phase). One hour later, one of the familiar objects was replaced with a novel object and the rats were placed once more in the apparatus with the same object and novel object for them to explore for $5 \mathrm{~min}$ (testing phase). A video camera mounted $100 \mathrm{~cm}$ above the center of the box was used to monitor and record the activity of rats. The discrimination ratio was defined as the time spent with the novel object to the total time spent exploring either object. Object presentation was randomized and counterbalanced across animals and groups. After each trial, the box and the objects were cleaned with a $75 \%$ ethanol solution in order to prevent olfactory cues from being perceived by other rats (Dong et al., 2017).

\section{Open field test (OFT)}

Motor deficits, locomotor activity and anxiety in animals were measure by OFT. The animals were carried to the test room in their home cages and allowed to acclimate for $30 \mathrm{~min}$ before the start of the locomotion test. The open field apparatus consisted of a clear Plexiglas box $(100 \times 100 \mathrm{~cm})$ with $30-\mathrm{cm}$-high walls and a white floor marked with a grid containing 16 squares. During a 5 -min observation period, the animals were placed in one corner of the apparatus facing the wall. All paths taken by the rat were recorded with a computer-controlled tracking system. The number of squares crossed with all four paws was determined from the video recording. The open field box was washed with a $70 \%$ ethanol before other animals were placed in it in order to prevent olfactory cues from being perceived by other rats (Adebiyi et al., 2016).

\section{Statistical analysis}


Table 2. Standardization of traditional herbal formulation (THF) according phenolic and felavonoids components.

\begin{tabular}{lccc}
\hline \multicolumn{1}{c}{ compounds } & $\begin{array}{c}\text { content } \\
(\boldsymbol{\mu} \mathbf{g} / \mathbf{g ~ D W})\end{array}$ & Retention time & Amax \\
\hline Ferulic acid & $123.1 \pm 0.4$ & 3.31 & 320 \\
Gallic acid & $2447.5 \pm 8.4$ & 6.42 & 270 \\
Caffeic acid & $58.6 \pm 0.1$ & 22.54 & 295 \\
Benzoic acid & $1301.0 \pm 0.5$ & 28.15 & 250 \\
Naringenin & $265.7 \pm 0.4$ & 30.30 & 285 \\
Rutin & $349.3 \pm 0.2$ & 33.44 & 360 \\
Quercetin & $134.7 \pm 0.6$ & 41.33 & 370 \\
Apigenin & $53.3 \pm 0.2$ & 52.22 & 336 \\
\hline
\end{tabular}

Results are mean of three replicates with standard errors (mean \pm SEM, $n=3), P<0.05$.

DW: dry weight

The SPSS software version 23.0 (SPSS Inc., Chicago, IL, U.S.A) and GraphPad Prism version 6.0 (GraphPad Software, San Diego, CA, USA) were applied for statistical analysis. All data analyzed by one-way analyses of variance (ANOVA) followed by Tukey's post hoc test. Results were considered significantly different if $P<0.05$.

\section{Results}

The effect of THF on body weight, FBS and insulin

At the end of the experiment, the final body weights and insulin in diabetic groups significantly decreased compared to the healthy control group $(P<0.001)$. Treatments (THF $300 \mathrm{mg} / \mathrm{kg}$ and $\mathrm{G}$ ) increased body weight and serum insulin level compared to the diabetic group $(P<0.001)$. FBS level was significantly increased in diabetic rats compared to the control group. In this regard, the results showed that the treatment with THF $200,300 \mathrm{mg} / \mathrm{kg}(P<0.001)$ or $\mathrm{G}$ $(P<0.05)$ significantly decreased FBS compared to the diabetic group (Table 1).

\section{The effect of THF on hippocampus oxidative toxic stress}

The comparison of the hippocampus oxidative stress parameters levels between all groups is summarized in Figure 2. As shown, the streptozotocin/ nicotinamide resulted in a notable decrease in the TAC and TTM, also increase in the MDA, TOS and OSI level $(P<0.001)$. The administration of THF could relieve these oxidative markers by a significant increase in the TAC (THF $300 \mathrm{mg} / \mathrm{kg}$ vs. D; $P<0.001$ ) and TTM (THF $300 \mathrm{mg} / \mathrm{kg}, P<0.01$ vs. D), and remarkable decrease in the MDA (THF $200 \mathrm{mg} / \mathrm{kg}$,
$P<0.01 ; 300 \mathrm{mg} / \mathrm{kg}, P<0.001$ vs. D), TOS (THF $300 \mathrm{mg} / \mathrm{kg}, P<0.001$ vs. D) and OSI (THF $300 \mathrm{mg} / \mathrm{kg}$, $P<0.001$, and $\mathrm{G}, P<0.01$ vs. D).

\section{The effect of THF on MWM, NORT and OFT}

As shown in Figure 3, diabetes significantly increased of the travelled distance (Fig. $3 \mathrm{~A}$ ) and escape latency (Fig. 3B) to find the hidden platform in the diabetic group at all three blocks compared to the control $(P<0.05)$. THF $300 \mathrm{mg} / \mathrm{kg}$ significantly decreased the distance and time to find the hidden platform in three blocks compared to diabetic group $(P<0.05)$. Diabetes resulted in significant decreases in the time spent (Fig. 3C) and travelled distance (Fig. 3D) in the target quadrant than those of the control group $(P<0.001)$. Animals of THF $300 \mathrm{mg} / \mathrm{kg}$ group significantly showed an increase in these parameters compared to the diabetes group $(P<0.001)$.

Effect of THF on NORT illustrated in Figure 4. There was a significant decrease in exploration time in the diabetes group compared to the control group $(P<0.001)$. THF $300 \mathrm{mg} / \mathrm{kg}$ returned this ration toward normal situation $(P<0.05)$.

Effects of THF on locomotion and anxiety-like behaviors in OFT are shown in Figure 4. There was a significant difference in the total distance moved (Fig. $4 \mathrm{~A})$ in the diabetes group compared to the control group $(P<0.05)$. Also these results confirmed that administration of THF $300 \mathrm{mg} / \mathrm{kg}$ significantly increased in the total distance moved compared to diabetic group $(P<0.05)$. In addition, velocity (Fig. 4B) between all groups did not significantly different.

Standardization of herbal formulation by RPHPLC-PDA 

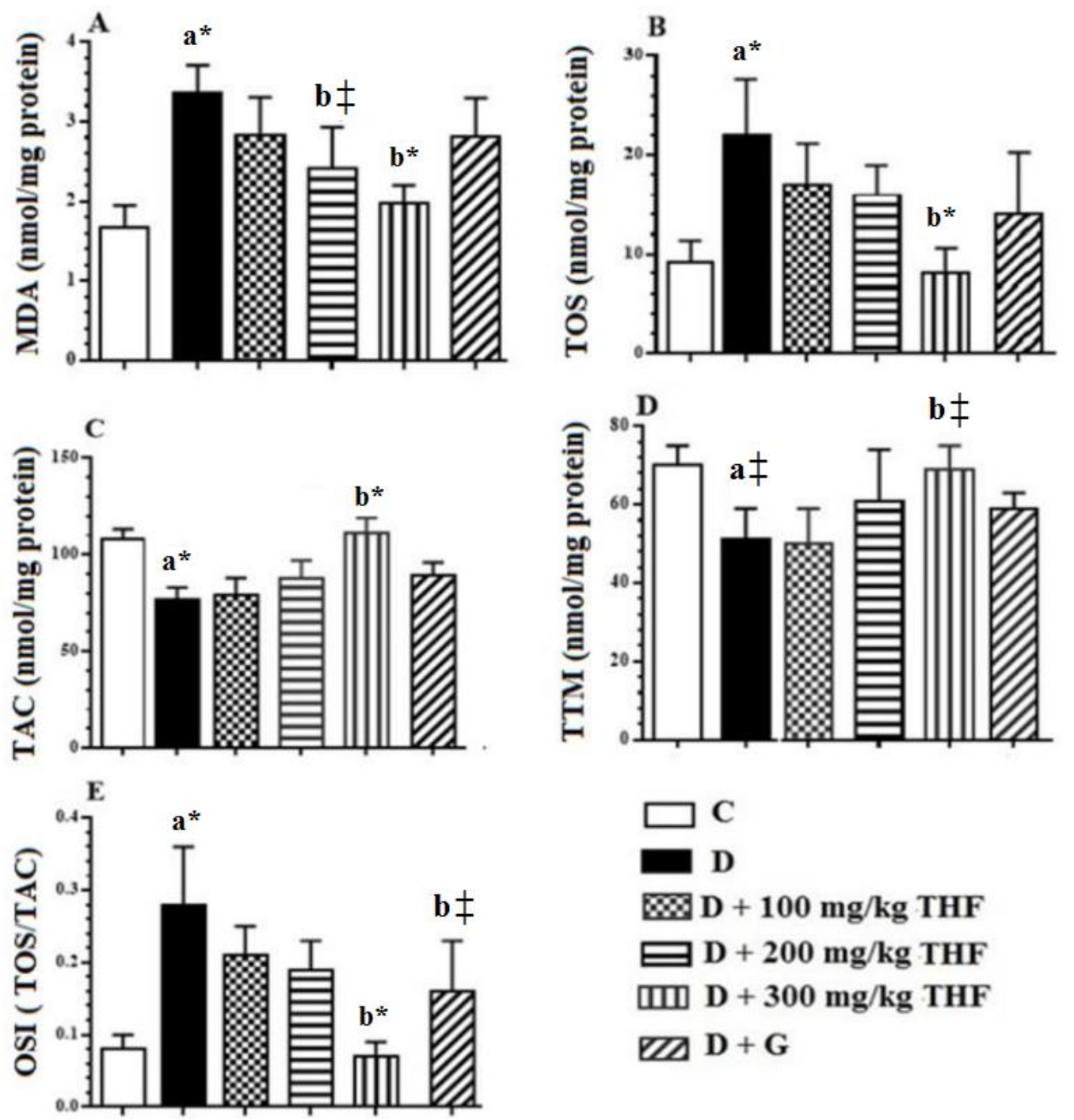

Fig.2. The effect of THF on hippocampus A: MDA, B: TOS, C: TAC, D: TTG, E: OSI. Results were expressed as mean $\pm S D$. C: control; D: diabetic; THF: traditional herbal formulation; G: glibenclamide 5mg/kg; MDA: malondialdehyde; TOS: total oxidant status; TAC: total antioxidant capacity; TTM: total thiol molecule; OSI: oxidative stress index. ${ }^{\mathrm{a}}$ significantly compared to the healthy control group. ${ }^{\mathrm{b}}$ significantly compared to the diabetic control group. ${ }^{\dagger} P<0.05,{ }^{\ddagger} P<0.01$ and ${ }^{*} P<0.001$.

Quantitative HPLC analysis indicated that gallic acid and benzoic acid (2447.5 to $1301.0 \mu \mathrm{g} / \mathrm{g}$ dry weight) were the main phenolic compounds in the herbal formulation. Also, the main flavonoids in herbal formulation were rutin and naringenin (349.3 to 265.7 $\mu \mathrm{g} / \mathrm{g}$ dry weight).

\section{Discussion}

Millions of people around the world suffer from DM and the prevalence of this disease continues to the rise. DM is an endocrine metabolic disorder defined by chronic hyperglycemia due to the deficiency in insulin secretion and peripheral insulin resistance. DM is one of the main causes of the prevalence of cognitive impairment (Arvanitakis et al., 2004). In this regard, the result of the present study showed learning and memory were impaired in T2DM rats. Also, our investigation showed that natural antioxidants THF decreased FBS, hippocampus oxidative stress and ameliorated behavioral ability in T2DM rats.

Insulin resistance are usually present in patients with classic T2DM. Insulin is the main glucoregulator that promotes the uptake of glucose by tissues and its subsequent use as an energy source (RodriguezAraujo et al., 2013). This hormone also performs unique functions within the central nervous system. Studies have demonstrated that the reduces insulin secretion or resistance to its function in the central nervous system creates various complications, 

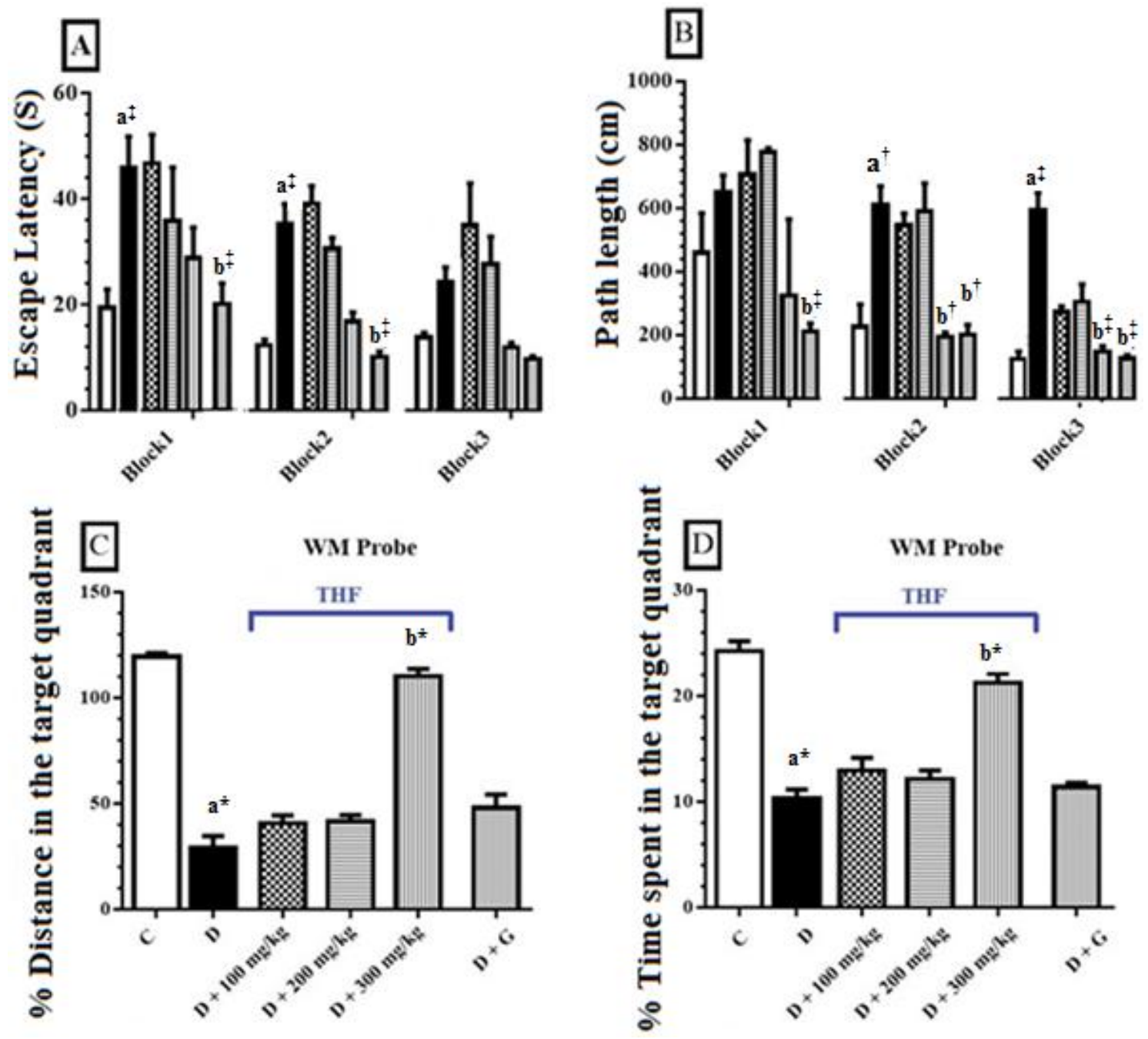

Fig.3. The effect of THF on spatial learning and memory in MWM test. C: control; D: diabetic; THF: traditional herbal formulation; G: glibenclamide $5 \mathrm{mg} / \mathrm{kg}$. Travelled distance (A) and escape latency (B) to find the hidden platform in three blocks. Time spent $(C)$ and travelled distance (D) in the target quadrant. Data are shown as mean $\pm S D$. ${ }^{a}$ significantly compared to the healthy control group. ${ }^{b}$ significantly compared to the diabetic control group. ${ }^{\dagger} P<0.05,{ }^{\ddagger} P<0.01$ and ${ }^{*} P<0.001$.

ranging from mild forgetfulness to Alzheimer's disease (Ma et al., 2015). In our experiment, serum insulin concentration was decreased in the diabetic group. Whereas, treatments with THF stimulated secretion of insulin, this factor may improve the memory and learning of the studied rats.

Also, our results showed that the FBS of diabetic control rats was significantly increased, but in diabetic treatment with THF (200 and $300 \mathrm{mg} / \mathrm{kg}$ ) and $\mathrm{G}$, it was significantly decreased. Studies show that hyperglycemic control appears to play an important role in determining the degree of cognitive decrements or dementia detected in patients with T2DM (Watson and Craft, 2003). Although hyperglycemia may not lead to cognitive decrements or dementia directly, it could certainly reduce the threshold for these disorders. Oxidative stress has been implicated as a contributor to both the onset and the progression of diabetes and its related complications. Hyperglycemia can lead to increase the production of oxidative stress markers, such as lipid peroxidation LPO and protein oxidation, with an accompanying reduction in antioxidant content, which have been suggested to be responsible for the induction of cognitive deficits often observed in diabetic rats (Comin et al., 2010; Fukui et al., 2002). In addition, hyperglycemia can contribute to the increased generation of advanced glycated end products (AGEs) (Wright et al., 2006). The cell surface receptor of AGEs (RAGE) can cause an increase in the production of intracellular oxidative stress (Ahmad et al., 2005). A diabetic animal with showed cognitive deterioration has been found to have over-expression of RAGEs in neurons in the 
A Novel Object Recognition Test

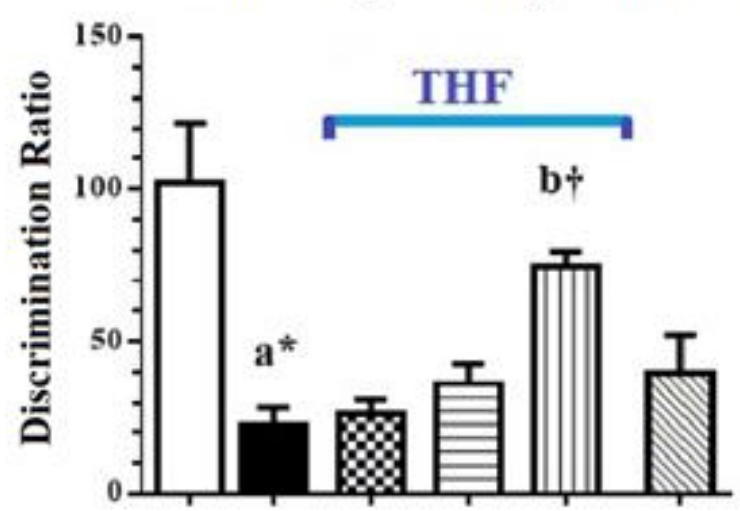

C

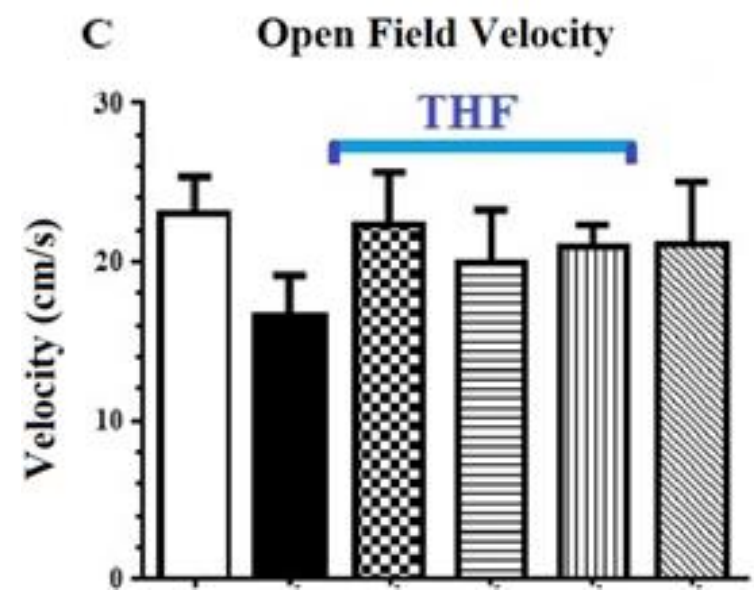

B

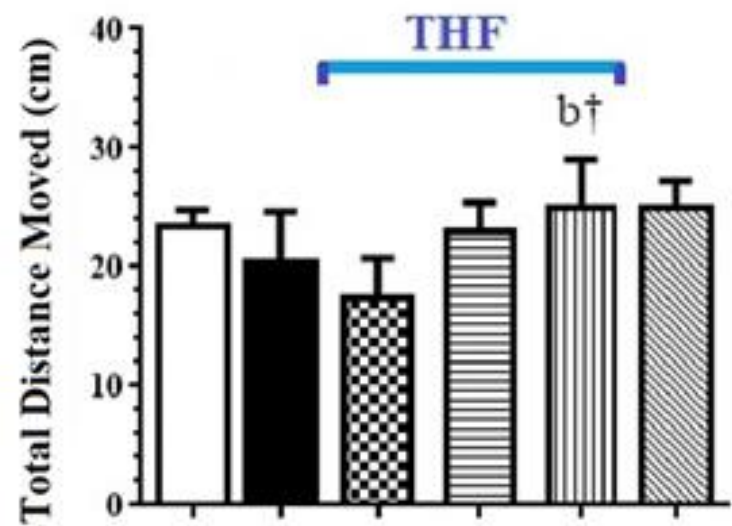

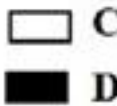

Q $\mathrm{D}+100 \mathrm{mg} / \mathrm{kg}$ THF

曰D $+200 \mathrm{mg} / \mathrm{kg}$ THF

سII $\mathrm{D}+300 \mathrm{mg} / \mathrm{kg}$ THF

ED $\mathbf{D}+\mathbf{G}$

Fig.4. The effect of THF on novel object recognition test, locomotion and anxiety-like behaviors in open field test. C: control; D: diabetic; THF: traditional herbal formulation; G: glibenclamide $5 \mathrm{mg} / \mathrm{kg}$. There was a significant decrease in exploration time in the diabetes group compared to the control group ( $A$, novel object recognition test). There was a significant difference in the total distance moved $(B)$ and no significant difference in the velocity $(C)$ between experimental groups. Data are shown as mean \pm SD. ${ }^{a}$ significantly compared to the healthy control group . ${ }^{b}$ significantly compared to the diabetic control aroup. ${ }^{\dagger} P<0.05$.

brain, suggesting the possible role of RAGEs in the progress of cerebral dysfunction (Toth et al., 2006). Therefore, insulin evaluation, FBS, and oxidative stress reduction may have a pivotal role in the improvement of diabetes-behavioral deficits. Results of our experiment demonstrate that serum FBS was more and insulin was decreased in the diabetic group. Whereas, THF treatments decreased serum FBS and increased the level of insulin.

The conditions of hyperglycemia in diabetics inhibits mitochondrial complex III and leads to the production of reactive oxygen spices in the hippocampus and other tissue. Increased oxidative stress in the brain seems to have an important role in the cognitive impairments caused by normal aging and neurodegenerative diseases (Ahmad et al., 2017). In this study, oxidative stress was investigated and the result indicated that the level of MDA, TOS and OSI in streptozotocin/nicotinamide diabetic rat increased and TTM and TAC level was decreased compared to the healthy group. Treatment with THF can reduce the level of MDA, TOS and OSI and increase the concentration of TTM and TAC compare to the diabetic group.

Numerous studies have demonstrated the beneficial effects of THM on cognitive dysfunction and dementia in diabetic condition (Ganji et al., 2017; Xu et al., 2013). The pharmacological mechanisms of the medicine herbs can be classified as reducing carbohydrate absorption, stimulating insulin secretion, increasing insulin sensitization, increasing peripheral glucose uptake, antioxidant effects and increasing the glycogenesis or inhibiting hepatic glycogenolysis (Wang et al., 2013). As mentioned reduced antioxidative levels and increased oxidative stress production is closely associated with the 
pathogenesis of diabetes and its complications such as neurodegenerative diseases. Studies have suggested the use of antioxidant supplementation to reduce oxidative stress and decelerate or prevent the development of disease-associated complications. Also, antioxidant properties of herbal medicines have been demonstrated in numerous studies (Sharifzadeh et al., 2017). Previous studies have investigated the antioxidant, antidiabetic and memory effects of the herbs used in the combination have been studied separately (Rezvani-Kamran et al., 2017; Shahidi and Yeo, 2018). Herbal formulation by RP-HPLC-PDA showed the THF contain ferulic acid, gallic acid, caffeic acid, benzoic acid, naringenin, rutin, quercetin and apigenin. The THF had an about $52 \%$ of gallic acid (3, 4, 5-trihydroxybenzoic acid). Pharmacological studies have demonstrated gallic acid is a potential antioxidant and neuroprotective effects from the family of phenolic compounds (Mansouri et al., 2013; Salehi et al., 2018). Gallic acid with three hydroxyl groups showed good antioxidant activity and can efficiently scavenge reactive oxygen specious (Velika and Kron, 2012). Also The THF had an about $23 \%$ of benzoic acid. Benzoic acid belongs to a group of phenolic compounds and showed antioxidant properties against different type of reactive oxygen specious and can prevent or decrease overproduction of them (Velika and Kron, 2012). The results of the present study indicate that the combined effects of these herbs are effective and may be a useful therapeutic option for memory disorders caused by diabetes.

In this study administration of a traditional herbal formulation has been shown to improve spatial learning and memory in MWM, NORT and OFT. Likewise, several studies have demonstrated that administration of traditional herbal formulation effect significantly greater than alone treatment on diabetes (Pirbalouti et al., 2012) and DM-associated cognitive impairment (Seto et al., 2015). Several mechanisms have been proposed as underlying the effect of traditional herbal formulation on DM-mediated cognitive dysfunction, such as through reduction of AGEs mediated neuroprotective and antiinflammation effect, and reducing cerebral amyloidbeta (Wang et al., 2013; Xu et al., 2013). In our study, antioxidant effects, increased insulin level, as well as hypoglycemic effects of traditional herbal formulation, may improve learning and memory in water mazes,
NORT and OFT. Pal et al. (2012) determined a similar modulatory effect about traditional extract protects animals against $\mathrm{CCl} 4$ induced renal oxidative impairments and necrotic cell death. These results clearly showed the antioxidative and improving learning and memory of traditional herbal formulation in T2DM induced cognitive dysfunction. Although these findings need to be confirmed in humans, it provides important preclinical data to support the potential benefits of traditional herbal formulation in preventing and slowing the progression and development of DM-associated cognitive dysfunction.

\section{Conclusion}

In summary, our results showed that the administration of the THF reduced oxidative stress, enhanced learning and memory and reversed the memory impairment induced by a T2DM. Although, further studies are essential to improve our understanding of the neurobiological mechanisms underlying the effects of the THF administration on learning and memory with the aim of to provide THF as a pharmacological agent to humans.

\section{Acknowledgments}

The authors sincerely thank the staff of Neurophysiology Research Center because of helping with this project. The study was funded by Hamadan University of Medical Sciences, Hamadan, Iran (project number: 9604202588).

\section{Conflict of interest}

No potential conflict of interest was reported by the authors.

\section{References}

Adebiyi OE, Olopade FE, Olopade JO, Olayemi FO. Behavioural studies on the ethanol leaf extract of Grewia carpinifolia in Wistar rats. Afr Health Sci 2016; 16: 339-46. https://doi.org/10.4314/ahs.v16i1.45

Ahmad FK, He Z, King GL. Molecular targets of diabetic cardiovascular complications. Curr Drug Targets 2005; 6: 487-94. https://doi.org/10.2174/1389450054021990

Ahmad W, ljaz B, Shabbiri K, Ahmed F, Rehman S. Oxidative toxicity in diabetes and Alzheimer's disease: mechanisms behind ROS/ RNS generation. J Biomed Sci 2017; 24: 76. https://doi.org/10.1186/s12929-0170379-z

Ardjmand A, Shahaboddin ME, Mazoochi T, Ghavipanjeh G. Ameliorative effects of cerebrolysin against isoproterenol-induced myocardial injury in male rats. 
Life Sci 2019; 227: 187-92. https://doi.org/10.1016/j.Ifs. 2019.04.056

Arvanitakis Z, Wilson RS, Bienias JL, Evans DA, Bennett DA. Diabetes mellitus and risk of Alzheimer disease and decline in cognitive function. Arch Neurol 2004; 61: 661 6. https://doi.org/10.1001/archneur.61.5.661

Asadbegi M, Yaghmaei P, Salehi I, Komaki A, EbrahimHabibi A. Investigation of thymol effect on learning and memory impairment induced by intrahippocampal injection of amyloid beta peptide in high fat diet- fed rats. Metab Brain Dis 2017; 32: 827-39. https://doi.org/ 10.1007/s11011-017-9960-0

Benzie IF, Strain JJ. The ferric reducing ability of plasma (FRAP) as a measure of "antioxidant power": the FRAP assay. Anal Biochem 1996; 239: 70-6. https://doi.org/ 10.1006/abio.1996.0292

Comin D, Gazarini L, Zanoni JN, Milani H, de Oliveira RM. Vitamin $\mathrm{E}$ improves learning performance and changes the expression of nitric oxide-producing neurons in the brains of diabetic rats. Behav Brain Res 2010; 210: 3845. https://doi.org/10.1016/j.bbr.2010.02.001

Dong J, Zhao J, Lin $\mathrm{Y}$, Liang $\mathrm{H}$, He X, Zheng $\mathrm{X}$, et al. Exercise improves recognition memory and synaptic plasticity in the prefrontal cortex for rats modelling vascular dementia. Neurol Res 2017; 40: 68-77. https://doi.org/10.1080/01616412.2017.1398389

Fukui K, Omoi NO, Hayasaka T, Shinnkai T, Suzuki S, Abe $\mathrm{K}$, et al. Cognitive impairment of rats caused by oxidative stress and aging, and its prevention by vitamin E. Ann NY Acad Sci 2002; 959: 275-84. https://doi.org/10.1111/j.1749-6632.2002.tb02099.x

Ganji A, Salehi I, Nazari M, Taheri M, Komaki A. Effects of Hypericum scabrum extract on learning and memory and oxidant/antioxidant status in rats fed a long-term high-fat diet. Metab Brain Dis 2017; 32: 1255-65. https://doi.org/10.1007/s11011-017-0022-4

Goli F, Karimi J, Khodadadi I, Tayebinia H, Kheiripour N, Hashemnia M, et al. Silymarin attenuates ELMO-1 and $\mathrm{KIM}-1$ expression and oxidative stress in the kidney of rats with type 2 diabetes. Indian J Clin Biochem 2019; 34: 172-9. https://doi.org/10.1007/s12291-018-0735-0

Guariguata L, Whiting DR, Hambleton I, Beagley J, Linnenkamp U, Shaw JE. Global estimates of diabetes prevalence for 2013 and projections for 2035. Diabetes Res Clin Pract 2014; 103: 137-49. https://doi.org/10. 1016/j.diabres.2013.11.002

Kheiripour N, Karimi J, Khodadadi I, Tavilani H, Goodarzi MT, Hashemnia M. Hepatoprotective effects of silymarin on liver injury via irisin upregulation and oxidative stress reduction in rats with type 2 diabetes. Iran J Med Sci 2019; 44: 108-117.

Kodl CT, Seaquist ER. Cognitive dysfunction and diabetes mellitus. Endocrine Rev 2008; 29: 494-511. https://doi.org/10.1210/er.2007-0034

Li WL, Zheng HC, Bukuru J, De Kimpe N. Natural medicines used in the traditional Chinese medical system for therapy of diabetes mellitus. J Ethnopharmacol 2004; 92: 1-21. https://doi.org/10.1016/ j.jep.2003.12.031
Ma L, Shao Z, Wang R, Zhao Z, Dong W, Zhang J, et al. Rosiglitazone improves learning and memory ability in rats with type 2 diabetes through the insulin signaling pathway. Am J Med Sci 2015; 350: 121-8. https://doi.org/10.1097/MAJ.0000000000000499

Mansouri MT, Farbood Y, Sameri MJ, Sarkaki A, Naghizadeh B, Rafeirad M. Neuroprotective effects of oral gallic acid against oxidative stress induced by 6 hydroxydopamine in rats. Food Chem 2013; 138: 102833. https://doi.org/10.1016/j.foodchem.2012.11.022

Pal PB, Pal S, Manna P, Sil PC. Traditional extract of Pithecellobium dulce fruits protects mice against $\mathrm{CCl} 4$ induced renal oxidative impairments and necrotic cell death. Pathophysiology 2012; 19: 101-14. https://doi.org/10.1016/j.pathophys.2012.02.001

Pirbalouti AG, Azizi S, Koohpayeh A. Healing potential of Iranian traditional medicinal plants on burn wounds in alloxan-induced diabetic rats. Rev bras farmacogn 2012; 22: 397-403. https://doi.org/10.1590/S0102$695 \times 2011005000183$

Rahimi R, Karimi J, Khodadadi I, Tayebinia H, Kheiripour N, Hashemnia $M$, et al. Silymarin ameliorates expression of urotensin II (U-II) and its receptor (UTR) and attenuates toxic oxidative stress in the heart of rats with type 2 diabetes. Biomed Pharmacother 2018; 101: 24450. https://doi.org/10.1016/j.biopha.2018.02.075

Ravan AP, Bahmani M, Basir HR, Salehi I, Oshaghi EA. Hepatoprotective effects of Vaccinium arctostaphylos against $\mathrm{CCl} 4$-induced acute liver injury in rats. J Basic Clin Physiol Pharmacol 2017; 28: 463-71. https://doi.org/10.1515/jbcpp-2016-0181

Rezvani-Kamran A, Salehi I, Shahidi S, Zarei M, Moradkhani S, Komaki A. Effects of the hydroalcoholic extract of Rosa damascena on learning and memory in male rats consuming a high-fat diet. Pharm Biol 2017; 55:

2065-73. https://doi.org/10.1080/13880209.2017.1362010

Rios JL, Francini F, Schinella GR. Natural products for the treatment of type 2 diabetes mellitus. Planta medica 2015; 81: 975-94. https://doi.org/10.1055/s-00351546131

Risner ME, Saunders AM, Altman JF, Ormandy GC, Craft $S$, Foley IM, et al. Efficacy of rosiglitazone in a genetically defined population with mild-to-moderate Alzheimer's disease. Pharmacogenomics J 2006; 6: 246-54. https://doi.org/10.1038/sj.tpj.6500369

Rodriguez-Araujo G, Nakagami $H$, Hayashi $H$, Mori $M$, Shiuchi T, Minokoshi $Y$, et al. Alpha-synuclein elicits glucose uptake and utilization in adipocytes through the Gab1/PI3K/Akt transduction pathway. Cell Mol Life Sci 2013; 70: 1123-33. https://doi.org/10.1007/s00018-0121198-8

Salehi A, Rabiei Z, Setorki M. Effect of gallic acid on chronic restraint stress-induced anxiety and memory loss in male BALB/c mice. Iran J Basic Med Sci 2018; 21: $1232-37$.

Seto SW, Yang GY, Kiat H, Bensoussan A, Kwan YW, Chang D. Diabetes mellitus, cognitive impairment, and traditional Chinese medicine. Int J Endocrinol 2015; 
2015. https://doi.org/10.1155/2015/810439

Shahidi F, Yeo J. Bioactivities of phenolics by focusing on suppression of chronic diseases: a review. Int J Mol Sci 2018; 19: 1573. https://doi.org/10.3390/ijms19061573

Sharifzadeh M, Ranjbar A, Hosseini A, Khanavi M. The effect of green tea extract on oxidative stress and spatial learning in streptozotocin-diabetic rats. Iran $\mathrm{J}$ Pharm Res 2017; 16: 201-9.

Shirwaikar A, Rajendran K, Barik R. Effect of aqueous bark extract of Garuga pinnata Roxb. in streptozotocinnicotinamide induced type-II diabetes mellitus. J Ethnopharmacol 2006; 107: 285-90. https://doi.org/10. 1016/j.jep.2006.03.012

Toth C, Schmidt AM, Tuor UI, Francis G, Foniok T, Brussee $V$, et al. Diabetes, leukoencephalopathy and rage. Neurobiol Dis 2006; 23: 445-61. https://doi.org/10.1016/ j.nbd.2006.03.015

Velika B, Kron I. Antioxidant properties of benzoic acid derivatives against superoxide radical. Free Rad Antiox 2012; 2: 62-7. https://doi.org/10.5530/ax.2012.4.11

Wang Z, Wang J, Chan P. Treating type 2 diabetes mellitus with traditional Chinese and Indian medicinal herbs. Evid Based Complementary Altern Med 2013; 2013. https://doi.org/10.1155/2013/343594

Watson GS, Craft $S$. The role of insulin resistance in the pathogenesis of Alzheimer's disease. CNS drugs 2003; 17: 27-45. https://doi.org/10.2165/00023210-200317010 $-00003$

Wright Jr E, Scism-Bacon JL, Glass LC. Oxidative stress in type 2 diabetes: the role of fasting and postprandial glycaemia. Int J Clin Pract 2006; 60: 308-14. https://doi.org/10.1111/j.1368-5031.2006.00825.x

Xu X, Guo L, Tian G. Diabetes cognitive impairments and the effect of traditional Chinese herbs. Evid Based Complementary Altern Med 2013; 2013. https://doi.org/10.1155/2013/649396

Zanganeh N, Siahpoushi E, Kheiripour N, Kazemi S, Goodarzi MT, Alikhani MY. Brucellosis causes alteration in trace elements and oxidative stress factors. Biol Trace Elem Res 2018; 182: 204-8. https://doi.org/10. 1007/s12011-017-1102-3

Zarrinkalam E, Ranjbar K, Salehi I, Kheiripour N, Komaki A. Resistance training and hawthorn extract ameliorate cognitive deficits in streptozotocin-induced diabetic rats. Biomed Pharmacother 2017; 97: 503-10. https://doi.org/ 10.1016/j.biopha.2017.10.138 